\title{
SIMETRISASI BENTUK KANONIK JORDAN
}

\section{Simmetrization of the Jordan Canonical Form}

\author{
Darlena $^{1 *}$, Ari Suparwanto ${ }^{2}$ \\ ${ }^{1}$ Prodi Manajemen Informatika Akademi Manajemen Komputer dan Informatika (AMKI) \\ ${ }^{1}$ Jln. Sutan Syahrir No.1, Ketapang, 78813, Kalimantan Barat, Indonesia \\ ${ }^{2}$ Departemen Matematika FMIPA Universitas Gadjah Mada \\ ${ }^{2}$ Sekip Utara Bulaksumur, Sinduadi, Kabupaten Sleman, Daerah Istimewa Yogyakarta, Indonesia
}

Corresponding author e-mail: ${ }^{1 *}$ darlena@mail.ugm.ac.id

\begin{abstract}
Abstrak
Jika polinomial karakteristik suatu operator linier $T$ terfaktorkan secara lengkap di lapangan skalar dari $T$ maka bentuk kanonik Jordan $J_{T}$ dapat diubah ke bentuk kanonik rasional $R_{T}$ dari $T$, demikian pula sebaliknya dan jika polinomial karakteristik suatu operator linier $T$ tidak terfaktorkan secara lengkap di lapangan skalar dari $T$ maka matriks bentuk kanonik rasional $R_{T}$ dari $T$ tetap dapat dibentuk namun tidak demikian dengan matriks bentuk kanonik Jordan $J_{T}$ dari $T$. Dalam kasus ini, matriks bentuk kanonik rasional $R_{T}$ dari $T$ dapat diubah menjadi matriks bentuk kanonik Jordan $J_{T}$ dari $T$ dengan memperluas lapangan skalar dari $T$ ke splitting field dari polinomial minimal $m_{T}(x)$ dari $T$, sehingga terbentuk matriks bentuk kanonik Jordan $J_{T}$ dari $T$ atas splitting field dari $m_{T}(x)$. Sebaliknya, untuk mengubah matriks bentuk kanonik Jordan $J_{T}$ dari $T$ atas splitting field dari $m_{T}(x)$ menjadi matriks bentuk kanonik rasional $R_{T}$, digunakan simetrisasi pada basis dekomposisi Jordan dari $T$ sehingga terbentuk basis dekomposisi siklik dari $T$ yang kemudian digunakan untuk membentuk matriks kanonik rasional $R_{T}$ dari $T$.
\end{abstract}

Kata Kunci : Bentuk kanonik rasional, Bentuk kanonik jordan, Splitting field, Simetrisasi.

\begin{abstract}
If the characteristic polynomial of a linear operator $T$ is completely factored in scalar field of $T$ then Jordan canonical form $J_{T}$ of $T$ can be converted to its rational canonical form $R_{T}$ of $T$, and vice versa. If the characteristic polynomial of linear operator $T$ is not completely factored in the scalar field of $T$, then the rational canonical form $R_{T}$ of $T$ can still be obtained but not its Jordan canonical form matrix $J_{T}$. In this case, the rational canonical form $R_{T}$ of $T$ can be converted to its Jordan canonical form by extending the scalar field of $T$ to Splitting Field of minimal polynomial $m_{T}(x)$ of $T$, thus forming the Jordan canonical form $J_{T}$ of $T$ over Splitting Field of $m_{T}(x)$. Conversely, converting the Jordan canonical form $J_{T}$ of $T$ over Splitting Field of $m_{T}(x)$ to its rational canonical form uses symmetrization on the Jordan decomposition basis of $T$ so as to form a cyclic decomposition basis of $T$ which is then used to form the rational canonical matrix of $T$.
\end{abstract}

Keywords: Rational canonical form, Jordan canonical form, Splitting field, Simmetrization.

Article info:

Submitted: $22^{\text {nd }}$ July 2020

Accepted: $13^{\text {th }}$ February 2021

How to cite this article:

Darlena, A. Suparwanto, "SIMETRISASI BENTUK KANONIK JORDAN", BAREKENG: J. Il. Mat. \& Ter., vol. 15, no. 1, pp. 015-028, Mar. 2021.

This work is licensed under a Creative Commons Attribution-ShareAlike 4.0 International License. Copyright (ㅇ 2021 Darlena, Ari Suparwanto 


\section{PENDAHULUAN}

Dalam tulisan ini, akan ditunjukkan bagaimana cara membentuk matriks bentuk kanonik Jordan dan matriks bentuk kanonik rasional dari suatu oprator linier $T$ dalam suatu ruang vektor $V$, dan bagaimana cara mengubah dari bentuk kanonik satu ke bentuk kanonik yang lainnya. Ruang vektor $V$ yang dimaksudkan adalah ruang vektor yang mempunyai dimensi berhingga. Untuk $\alpha \in V, Z(\alpha, T)$ menotasikan subruang siklik dari $V$ yang dibangun oleh $\left\{\alpha, T(\alpha), T^{2}(\alpha), \ldots\right\}$ (di domain dari $T$ ). Pembangun $f(x):=x^{k}+a_{k-1} x^{k-1}+$ $\cdots+a_{1} x+a_{0}$ dari ideal utama yang berisi polinomial-polinomial $g(x) \in \mathbb{F}[x]$ yang memenuhi $g(T)(\alpha)=$ 0 disebut polinomial $T$-annihilator dari $\alpha$ yang dinotasikan dengan $m_{\alpha}(x)$. Dimensi dari ruang $Z(\alpha, T)$ ditandai oleh derajat dari polinomial $m_{\alpha}(x)$ [2]. Di dalam Hoffman and Kunze [2] juga dijelaskan cara pembentukan matriks bentuk kanonik Jordan dan matriks bentuk kanonik rasional dari suatu operator linier $T$ yang dijabarkan sebagai berikut. Jika terdapat vektor-vektor tak-nol $\alpha_{1}, \alpha_{2}, \ldots, \alpha_{r} \in V$ sedemikian sehingga

$$
V=Z\left(\alpha_{1}, T\right) \oplus Z\left(\alpha_{2}, T\right) \oplus \ldots \oplus Z\left(\alpha_{r}, T\right)
$$

maka bentuk (1) disebut dekomposisi siklik dari $V$ terhadap $T$. Misalkan $\mathcal{A}=\cup_{i=1}^{r} \mathcal{A}_{i}$ dengan $\mathcal{A}_{i}$ adalah basis siklik dari ruang $Z\left(\alpha_{i}, T\right)$, maka matriks representasi dari $T$ terhadap $\mathcal{A}$ berbentuk matriks bentuk kanonik rasional, yaitu

$$
R_{T}=\left[\begin{array}{cccc}
C\left[m_{\alpha_{1}}(x)\right] & 0 & \cdots & 0 \\
0 & C\left[m_{\alpha_{1}}(x)\right] & \cdots & 0 \\
\vdots & \vdots & \ddots & \vdots \\
0 & 0 & \cdots & C\left[m_{\alpha_{r}}(x)\right]
\end{array}\right]
$$

dengan $C\left[m_{\alpha_{i}}(x)\right]$ adalah matriks kompanion dari polinomial $m_{\alpha}(x)$ untuk $i=1,2, \ldots, r$. Misalkan $M_{T}(x)=$ $p_{1}(x)^{t_{1}} \ldots p_{k}(x)^{t_{k}}$ adalah polinomial minimal dari $T$, maka

$$
V=\operatorname{Ker}\left(p_{1}(T)^{t_{1}}\right) \oplus \ldots \oplus \operatorname{Ker}\left(P_{r}(T)^{t_{r}}\right)
$$

Bentuk (2) disebut dekomposisi primer dari $V$. Lebih lanjut, dengan mendekomposisi siklikkan masing-masing ruang $\operatorname{Ker}\left(p_{i}(T)^{t_{i}}\right)$ dan menggabungkan basis dari masing-masing ruang tersebut, diperoleh suatu basis $\mathcal{B}$ sedemikian sehingga matriks representasi dari $T$ terhadap basis $\mathcal{B}$ berbentuk matriks bentuk kanonik Jordan, yaitu

$$
[T]_{\mathcal{B}}=\left[\begin{array}{cccc}
A_{1} & 0 & \cdots & 0 \\
0 & A_{2} & \cdots & 0 \\
\vdots & \vdots & \ddots & \vdots \\
0 & 0 & \cdots & A_{k}
\end{array}\right]
$$

dengan $A_{i}$ adalah matriks segmen Jordan yang bersesuai dengan nilai eigen $\lambda_{i}$, untuk $i=1, \ldots, k$. Orde dari matriks $A_{i}$ menandakan multiplisitas aljabar dari nilai eigen $\lambda_{i}$ pada polinomial karakteristik $p_{T}(x)$ dari $T$ dan orde blok Jordan terbesar pada matriks segmen Jordan $A_{i}$ menandakan multiplisitas aljabar dari nilai eigen $\lambda_{i}$ pada polinomial minimal $m_{T}(x)$ dari $T$. Sebagai perbandingan dalam memahami pembentukan matriks bentuk kanonik Jordan, di dalam Weintraub [5] juga dijelaskan cara pembentukan matriks bentuk kanonik Jordan dari suatu operator linier dengan menggunakan proses diagonalisasi.

Berdasarkan uraian di atas, pembentukan matriks bentuk kanonik Jordan $J_{T}$ dari $T$ sangat bergantung pada polinomial karakteristik $p_{T}(x)$ dari $T$, sedangkan pembentukan matriks bentuk kanonik rasional $R_{T}$ dari $T$ tidak bergantung pada polinomial karakteristik $p_{T}(x)$ dari $T$. Namun, jika dilihat dari proses pembentukannya, maka matriks bentuk kanonik Jordan $J_{T}$ dan matriks bentuk kanonik rasional $R_{T}$ dari $T$ sama-sama menggunakan suatu basis dari ruang vektor $V$ yang diperoleh melalui suatu dekomposisi dari ruang vektor $V$ tersebut. Namun dalam kasus tertentu, yaitu ketika polinomial karakteristik $p_{T}(x)$ dari $T$ taktereduksi atau tidak terfaktorkan secara lengkap di lapangan $\mathbb{F}$, maka akan dihasilkan suatu matriks bentuk kanonik Jordan $J_{T}$ dari $T$ atas lapangan perluasan dari $\mathbb{F}$, yaitu splitting field dari polinomial minimal $m_{T}(x)$ dari $T$. Kajian tentang lapangan perluasan atau lebih khususnya splitting field dari suatu polinomial dapat dilihat pada Fraleigh [1]. 
Berdasarkan Radjabalipour (2013) [3] dan Radjabalipour (2017) [4], Matriks bentuk kanonik Jordan $J_{T}$ atas lapangan perluasan dari $\mathbb{F}$ tidak dapat diubah ke bentuk kanonik rasional $R_{T}$ dari $T$ atas lapangan $\mathbb{F}$, karena dari perluasan suatu lapangan $\mathbb{F}$, tidak dapat diperoleh informasi tentang lapangan $\mathbb{F}$. Tetapi, matriks bentuk kanonik Jordan $J_{T}$ dari $T$ tersebut dapat diubah menjadi matriks bentuk kanonik rasional $R_{T}$ dengan menggunakan simetrisasi, yaitu dengan mengubah basis Jordan yang simetris dari $T$ menjadi basis siklik dari $T$ atas lapangan $\mathbb{F}$.

Oleh karena itu, perlu dikaji tentang hubungan antara matriks kanonik Jordan $J_{T}$ dan matriks kanonik rasional $R_{T}$ dalam hal cara memperoleh bentuk kanonik rasional dari bentuk kanonik Jordan dan sebaliknya, baik ketika polinomial karakteristik $p_{T}(x)$ dari $T$ terfaktorkan secara lengkap atau tidak di lapangan skalar dari $T$.

\section{METODE PENELITIAN}

Adapun langkah-langkah penelitian dalam penelitian ini adalah sebagai berikut:

1. Mempelajari cara pembentukan dekomposisi primer dan dekomposisi siklik ruang vektor.

2. Mempelajari cara pembetukan matriks bentuk kanonik rasional melalui dekomposisi siklik ruang vektor dan cara pembetukan matriks bentuk kanonik Jordan melalui dekomposisi primer dan dekomposisi siklik ruang vektor.

3. Mencari dan mempelajari contoh-contoh matriks bentuk kanonik rasional dan matriks bentuk kanonik Jordan dari suatu operator linier.

4. Menyelidiki keterkaitan blok-blok rasional pada matriks $R_{T}$ dan blok-blok Jordan pada matriks $J_{T}$ dengan polinomial minimal $T$ dan polinomial karakteristik $p_{T}$ dari $T$.

5. Mempelajari sifat-sifat lapangan perluasan dari suatu lapangan $\mathbb{F}$, lebih khususnya splitting field dari suatu polinomial yang tidak terfaktorkan secara lengkap di lapangan $\mathbb{F}$.

6. Mempelajari perluasan kanonik $T_{\mathbb{L}}$ dari operator linier $T_{\mathbb{F}}$ dan $V_{\mathbb{L}}$ dari ruang vektor $V_{\mathbb{F}}$ dengan $\mathbb{L}$ adalah suatu perluasan dari $\mathbb{F}$. Hal ini digunakan untuk menyelidiki basis $T_{\mathbb{L}}$-siklik dari $T$ ketika polinomial minimal dari $T$ tidak terfaktorkan secara lengkap di lapangan $\mathbb{F}$.

7. Mempelajari suatu automorfisma dari lapangan $\mathbb{L}$ yang akan digunakan untuk mengubah elemen-elemen di lapangan perluasan dari $\mathbb{L}$ menjadi elemen-elemen di lapangan $\mathbb{F}$. Kemudian hal ini akan digunakan untuk mengubah vektor-vektor basis $T_{\mathbb{L}}$-siklik primer dari $V$ dengan elemen di lapangan $\mathbb{L}$ menjadi vektor-vektor baru.

8. Mempelajari penggunaan vektor-vektor baru tersebut untuk membentuk basis $T_{\mathbb{F}}$-siklik dari $V$ dengan elemen di lapangan $\mathbb{F}$ sehingga dengan menggunakan basis $T_{\mathbb{F}}$-siklik tersebut diperoleh suatu dekomposisi siklik dari $V$.

\section{HASIL DAN PEMBAHASAN}

\subsection{Pengubahan Bentuk Kanonik Rasional Menjadi Bentuk Kanonik Jordan}

Misalkan $T$ adalah operator linear pada suatu ruang vektor $V$ berdimensi $n$ atas lapangan $\mathbb{F}$. Dari bentuk kanonik rasional dari $T$ diperoleh polinomial minimal $m_{T}(x)$ dari $T$. Dalam kasus $m_{T}(x)$ terfaktorkan secara lengkap di $\mathbb{F}$, diperoleh seluruh nilai eigen dari $T$. Dengan demikian, matriks bentuk kanonik Jordan dari $T$ dapat dibentuk dengan menggunakan ruang-ruang eigen dari $T$.

Contoh 1. Misalkan

$$
R_{T}=\left[\begin{array}{cc}
0 & 2 \\
1 & -1
\end{array}\right]
$$

adalah matriks bentuk kanonik rasional dari suatu operator linier $T$ pada ruang vektor $\mathbb{R}^{2}$. 
Berdasarkan proses pembentukan matriks bentuk kanonik rasional $R_{T}$ dari suatu operator linier $T$ yang diberikan pada bagian pendahuluan dari paper ini, diperoleh bahwa blok pertama dari $R_{T}$ merupakan matriks kompanion dari suatu polinomial $m_{\alpha}(x)$, yaitu

$$
m_{\alpha}(x)=x^{2}+x-2 .
$$

Berdasarkan akibat Teorema Dekomposisi Siklik (lihat Hoffman and Kunze [2], hal 237), diperoleh bahwa $m_{\alpha}(x)$ adalah polinomial minimal dari $T$, yaitu

$$
m_{T}(x)=x^{2}+x-2=(x+2)(x-1)
$$

Berdasarkan Teorema Dekomposisi Primer (lihat Hoffman and Kunze [2], hal 220), diperoleh

$$
V=\operatorname{Ker}(T+2 I) \oplus \operatorname{Ker}(T-I)
$$

Selajutnya didefinisikan operator nilpoten $N_{1}$ pada $\operatorname{Ker}(T+2 I)$ dan $N_{2}$ pada $\operatorname{Ker}(T-I)$, dengan $N_{1}=T+$ $2 I$ dan $N_{2}=T-I$. Berdasarkan Teorema Dekomposisi Siklik (lihat Hoffman and Kunze [2], hal 233), terdapat vektor-vektor tak-nol $\gamma_{1} \in \operatorname{Ker}(T+2 I)$ dan $\gamma_{2} \in \operatorname{Ker}(T-I)$ sedemikian sehingga

$$
\operatorname{Ker}(T+2 I)=Z\left(\gamma_{1} ; N_{1}\right) \text { dan } \operatorname{Ker}(T-I)=Z\left(\gamma_{2} ; N_{2}\right)
$$

Diambil $\gamma_{1}=\left[\begin{array}{c}-1 \\ 1\end{array}\right]$, dan $\gamma_{2}=\left[\begin{array}{l}2 \\ 1\end{array}\right]$. Dapat ditunjukan dengan mudah bahwa $\mathcal{B}=\left\{\gamma_{1}, \gamma_{2}\right\}$ membentuk basis untuk $\mathbb{R}^{2}$. Diperhatikan,

$$
\begin{gathered}
{\left[T\left(\gamma_{1}\right)\right]=\left[T\left(\left[\begin{array}{c}
-1 \\
1
\end{array}\right]\right)\right]=\left[\begin{array}{cc}
0 & 2 \\
1 & -1
\end{array}\right]\left[\begin{array}{c}
-1 \\
1
\end{array}\right]=\left[\begin{array}{c}
2 \\
-2
\end{array}\right]=(-2)\left[\begin{array}{c}
-1 \\
1
\end{array}\right]+(0)\left[\begin{array}{l}
2 \\
1
\end{array}\right]} \\
{\left[T\left(\gamma_{2}\right)\right]=\left[T\left(\left[\begin{array}{l}
2 \\
1
\end{array}\right]\right)\right]=\left[\begin{array}{cc}
0 & 2 \\
1 & -1
\end{array}\right]\left[\begin{array}{l}
2 \\
1
\end{array}\right]=\left[\begin{array}{l}
2 \\
1
\end{array}\right]=(0)\left[\begin{array}{c}
-1 \\
1
\end{array}\right]+(1)\left[\begin{array}{l}
2 \\
1
\end{array}\right]}
\end{gathered}
$$

Diperoleh, $\left[T\left(\gamma_{1}\right)\right]_{\mathcal{B}}=\left[\begin{array}{c}-2 \\ 0\end{array}\right]$ dan $\left[T\left(\gamma_{2}\right)\right]_{\mathcal{B}}=\left[\begin{array}{l}0 \\ 1\end{array}\right]$, sehingga

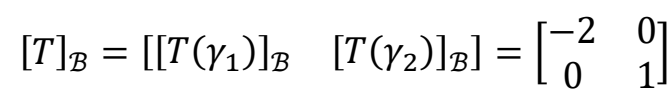

yang merupakan bentuk kanonik Jordan dari $T$.

Dalam kasus polinomial minimal dari $T$ tidak terfaktokan secara lengkap pada $\mathbb{F}[x]$, lapangan $\mathbb{F}$ diperluas ke splitting field $\mathbb{L}$ dari $m_{T}(x)$ sehingga $m_{T}(x)$ terfaktorkan secara lengkap di $\mathbb{L}$. Dengan langkah yang analog, diperoleh seluruh nilai eigen dari $T$ di lapangan $\mathbb{L}$, sehingga terbentuk matriks bentuk kanonik Jordan dari $T$ atas lapangan $\mathbb{L}$.

Contoh 2. Misalkan $T$ adalah operator linear pada $\mathbb{R}^{2}$ dengan matriks bentuk kanonik rasionalnya adalah

$$
\left[\begin{array}{cc}
0 & -1 \\
1 & 0
\end{array}\right]
$$

Polinomial minimal dari $T$ adalah $p_{T}(x)=m_{T}(x)=x^{2}+1$, yang merupakan polinomial tak-tereduksi pada lapangan $\mathbb{R}$. Oleh karena itu, polinomial tersebut akan dipandang sebagai suatu polinomial atas lapangan $\mathbb{C}$ (splitting field dari $m_{T}(x)$ ), menjadi $p_{T}(x)=m_{T}(x)=(x-i)(x+i)$. Diperoleh vektor eigen yang bersesuaian dengan masing-masing nilai eigen adalah $\gamma_{1}=\left[\begin{array}{c}1 \\ -i\end{array}\right]$, dan $\gamma_{2}=\left[\begin{array}{l}1 \\ i\end{array}\right]$. Dapat ditunjukkan bahwa $\mathcal{B}=\left\{\gamma_{1}, \gamma_{2}\right\}$ membentuk basis dari $\mathbb{C}^{2}$. Melalui suatu perhitungan, diperoleh matriks representasi dari $T$ terhadap basis $\mathcal{B}$ adalah

$$
[T]_{\mathcal{B}}=\left[\begin{array}{cc}
i & 0 \\
0 & -i
\end{array}\right]
$$


yang merupakan bentuk kanonik Jordan dari $T$ atas lapangan $\mathbb{C}$.

\subsection{Pengubahan Bentuk Kanonik Jordan Menjadi Bentuk Kanonik Rational}

Misalkan $T$ adalah suatu operator linear pada suatu ruang vektor $V$ berdimensi $n$ atas lapangan $\mathbb{F}$. Dari bentuk kanonik Jordan dari $T$ diperoleh polinomial minimal $m_{T}(x)$ dan polinomial karakteristik $p_{T}(x)$ dari $T$. Dalam kasus $p_{T}(x)$ terfaktorkan secara lengkap di $\mathbb{F}$, diperoleh seluruh nilai eigen dari $T$ dan dekomposisi primer dari $V$, yaitu

$$
V=V_{1} \oplus V_{2} \oplus \ldots \oplus V_{k}
$$

dengan $V_{1}, \ldots V_{k}$ adalah ruang eigen dari $T$. Untuk sebarang ventor tak nol $\alpha$ di $V$, terdapat dengan tunggal vektor-vektor tak-nol $\beta_{1}, \ldots, \beta_{k}$ dengan $\beta_{i} \in V_{i}$ sedemikian sehingga $\alpha=\beta_{1}+\cdots+\beta_{k}$. Karena $T\left(\beta_{i}\right)=$ $\lambda_{i} \beta_{i}$, maka

$$
f(T)(\alpha)=f(T)\left(\beta_{1}+\cdots+\beta_{k}\right)=f\left(\lambda_{1}\right) \beta_{1}+\cdots+f\left(\lambda_{k}\right) \beta_{k}
$$

untuk setiap polinomial $f(x) \in \mathbb{F}[x]$. Karena $f(T)(\alpha)$ adalah sebarang elemen di $Z(\alpha, T)$, berdasarkan (3), $Z(\alpha, T)$ dibangun oleh vektor-vektor $\beta_{1}, \ldots, \beta_{k}$ dan $f(T)(\alpha)=0$ jika dan hanya jika $f\left(\lambda_{i}\right) \beta_{i}=0$ untuk setiap $i=1, \ldots, k$. Oleh karena itu $T$-annihilator dari $\alpha$ adalah $m_{\alpha}(x)=\prod_{i=1}^{k}\left(x-\lambda_{i}\right)$. Misalkan $\mathcal{B}_{i}=\left\{\beta_{1}^{i}, \ldots, \beta_{d}^{i}\right\}$ adalah suatu basis dari $V_{i}$ dan misalkan $r=\operatorname{maks}\left\{d_{i} \mid i=1, \ldots, r\right\}$. Didefinisikan vektor-vektor tak-nol $\alpha_{1}, \ldots \alpha_{r}$ dengan $\alpha_{j}=\sum_{d_{i} \geq j} \beta_{j}^{i}$ untuk $j=1, \ldots, r$. Polinomial $T$-annihilator dari $\alpha_{j}$ adalah

$$
m_{\alpha_{j}}(x)=\prod_{d_{i} \geq j}\left(x-\lambda_{i}\right) .
$$

Diambil sebarang $g(T)\left(\alpha_{j}\right) \in Z\left(\alpha_{j} ; T\right)$, diperoleh

$$
g(T)\left(\alpha_{j}\right)=g(T)\left(\sum_{d_{i} \geq j} \beta_{j}^{i}\right)=\sum_{d_{i} \geq j} g(T)\left(\beta_{j}^{i}\right)=\sum_{d_{i} \geq j} g\left(\lambda_{i}\right)\left(\beta_{j}^{i}\right) .
$$

Jadi $Z\left(\alpha_{j} ; T\right)$ dibangun oleh vektor-vektor $\left\{\beta_{i}^{j} \mid d_{i} \geq j\right\}$ untuk $i=1, \ldots, r$. Karena masing-masing $\beta_{i}^{j}$ berada pada satu dan hanya salah satu dari ruang $Z\left(\alpha_{1} ; T\right), \ldots Z\left(\alpha_{r} ; T\right)$ dan $\mathcal{B}=\cup_{i=1}^{r} \mathcal{B}_{i}$ adalah basis dari $V$ maka diperoleh $V=Z\left(\alpha_{1} ; T\right) \oplus \ldots \oplus Z\left(\alpha_{r} ; T\right)$ dan $m_{\alpha_{j+1}}(x)$ membagi $m_{\alpha_{j}}(x)$ untuk $j=1, \ldots, r$. Dengan demikian, terdapat suatu basis $\mathcal{B}^{*}$ dari $V$ sedemikian sehingga matriks representasi dari $T$ terhadap basis $\mathcal{B}^{*}$ berbentuk kanonik rasional.berbentuk kanonik rasional.

Contoh 3. Misalkan $T$ adalah suatu operator linier pada ruang vektor $\mathbb{R}^{3}$ dengan matriks bentuk kanonik Jordannya adalah

$$
J_{T}=\left[\begin{array}{lll}
2 & 0 & 0 \\
0 & 2 & 0 \\
0 & 0 & 6
\end{array}\right]
$$

Dari matriks $J_{T}$ tersebut diperoleh polinomial karakteristik dan polinomial minimal dari $T$ adalah

$$
p_{T}(x)=(x-2)^{2}(x-6)
$$

dan

$$
m_{T}(x)=(x-2)(x-6)
$$

Berdasarkan Teorema Dekomposisi Primer, diperoleh

$$
V=W_{1} \oplus W_{2}
$$

dengan $W_{1}$ adalah ruang null dari $T-2 I$ dan $W_{2}$ adalah ruang null dari $T-6 I$. Ruang eigen $E(2)=W_{1}$ berdimensi 2 dengan basis $\left[\begin{array}{c}1 \\ -1 \\ 0\end{array}\right]$ dan $\left[\begin{array}{c}0 \\ 1 \\ -1\end{array}\right]$, dan ruang eigen $E(6)=W_{2}$ berdimensi 1 dengan basis $\left[\begin{array}{l}1 \\ 2 \\ 1\end{array}\right]$. Selanjutnya didefinisikan operator nilpoten $N_{i}$ pada masing-masing $W_{i}$, yaitu

$$
N_{1}=T-2 I \text { dan } N_{2}=T-6 I \text {. }
$$


dengan $m_{N_{1}}(x)=x$ dan $m_{N_{2}}(x)=x$. Berdasarkan Teorema Dekomposisi Siklik, terdapat vektor-vektor tak-nol $\gamma_{1}, \gamma_{2} \in W_{1}$ dan nol $\gamma_{3} \in W_{2}$, sedemikian sehingga

$$
W_{1}=Z\left(\gamma_{1} ; N_{1}\right) \oplus Z\left(\gamma_{2} ; N_{1}\right) \text { dan } W_{2}=Z\left(\gamma_{3} ; N_{2}\right) \text {. }
$$

Terkait dengan basis dari $W_{1}$ dan $W_{2}$ yang diperoleh sebelumnya, diambil

$$
\gamma_{1}=\left[\begin{array}{c}
1 \\
-1 \\
0
\end{array}\right], \gamma_{2}=\left[\begin{array}{c}
0 \\
1 \\
-1
\end{array}\right], \gamma_{3}=\left[\begin{array}{l}
1 \\
2 \\
1
\end{array}\right]
$$

dan $\mathcal{B}=\left\{\gamma_{1}, \gamma_{2}, \gamma_{3}\right\}$ membentuk basis dari $V$. Karena $r=2$, selanjutnya didefinisikan vektor-vektor taknol $\alpha_{1}, \alpha_{2} \in V$ dengan

$$
\alpha_{1}=\left[\begin{array}{c}
1 \\
-1 \\
0
\end{array}\right]+\left[\begin{array}{l}
1 \\
2 \\
1
\end{array}\right]=\left[\begin{array}{c}
2 \\
1 \\
-1
\end{array}\right]
$$

sedemikian sehingga

$$
V=Z\left(\alpha_{1} ; T\right) \oplus Z\left(\alpha_{2} ; T\right)
$$

dan $\mathcal{B}^{*}=\left\{\alpha_{1}, T\left(\alpha_{1}\right), \alpha_{2}\right\}$ membentuk basis dari $V$. Melalui proses perhitungan diperoleh matriks representasi dari $T$ terhadap basis $\mathcal{B}^{*}$ adalah

$$
[T]_{\mathcal{B}^{*}}=\left[\begin{array}{ccc}
0 & -12 & 0 \\
1 & 8 & 0 \\
0 & 0 & 2
\end{array}\right]
$$

yang berbentuk kanonik rasional.

Dalam kasus $p_{T}(x)$ tidak terfaktorkan secara lengkap di $\mathbb{F}$, artinya matriks bentuk kanonik Jordan dari $T$ adalah matriks atas lapangan perluasan $\mathbb{L}$ dari $\mathbb{F}$. Untuk pengubahan matriks bentuk kanonik Jordan $J_{T}$ atas $\mathbb{L}$ menjadi matriks bentuk kanonik rasioanal $R_{T}$ atas lapangan $\mathbb{F}$, akan digunakan simetrisasi pada basis dekomposisi siklik. Simetrisasi pada basis dekomposisi Jordan yang dimaksudkan adalah memilih suatu basis dekomposisi Jordan yang memenuhi syarat simetris.

Selanjutnya diberikan definisi ruang vektor yang berisi fungsi-fungsi yang mempunyai support berhingga.

Definisi 4. Misalkan $B$ adalah suatu himpunan dan $\mathbb{F}$ adalah suatu lapangan. Didefinisikan $\left(\mathbb{F}^{\mathbf{B}}\right)_{0}$ adalah ruang vektor yang berisi fungsi-fungsi dari B ke $\mathbb{F}$ yang mempunyai support berhingga.

Berdasarkan Definisi 4, himpunan $\left(\mathbb{F}^{\boldsymbol{B}}\right)_{0}$ dapat ditulis sebagai

$$
\left(\mathbb{F}^{B}\right)_{0}=\{f: B \rightarrow \mathbb{L} \mid \operatorname{supp}(f)<\infty\} .
$$

Untuk suatu $a \in B, f(a) \in \mathbb{L}$ dimana $\mathbb{L}$ adalah suatu lapangan perluasan dari $\mathbb{F}$. Fungsi-fungsi $f \in\left(\mathbb{F}^{B}\right)_{0}$ dapat ditulis sebagai $f(a)=c_{a} \in \mathbb{L}$ untuk suatu $a \in B$. Oleh karena itu, fungsi $f$ dapat ditulis kembali menjadi $(f(a))_{a \in B}=\left(c_{a}\right)_{a \in B}$, untuk suatu $a \in B$.

Teorema berikut menjelaskan tentang pembentukan basis dari ruang vektor $\left(\mathbb{F}^{B}\right)_{0}$.

Teorema 5. Misalkan $B$ adalah himpunan dengan kardinalitas $k$. Jika fungsi-fungsi $\delta_{b} \in\left(\mathbb{F}^{B}\right)_{0}$ untuk setiap $b \in B$ dengan

$$
\delta_{b}(x)= \begin{cases}1, & x=b \\ 0, & x \neq b\end{cases}
$$

maka himpunan $\mathcal{B}=\left\{\delta_{b} \mid b \in B\right\}$ membentuk basis untuk $\left(\mathbb{F}^{B}\right)_{0}$. Selanjutnya basis $\mathcal{B}$ disebut basis standar untuk $\left(\mathbb{F}^{B}\right)_{0}$. Oleh karena itu, $\operatorname{dim}\left(\mathbb{F}^{B}\right)_{0}=|B|$.

Bukti. Diketahui $|B|=k$ dan $\left(\mathbb{F}^{B}\right)_{0}$ adalah ruang vektor yang berisi fungsi-fungsi dari $B$ ke $\mathbb{F}$ yang mempunyai support berhingga. Diberikan himpunan bagian $\mathcal{B}=\left\{\delta_{b} \mid b \in B\right\}$ dari $\left(\mathbb{F}^{B}\right)_{0}$ yang didefinisikan untuk semua $b \in B$ dengan 


$$
\delta_{b}(x)= \begin{cases}1, & x=b \\ 0, & x \neq b\end{cases}
$$

Akan ditunjukkan $\mathcal{B}=\left\{\delta_{b} \mid b \in B\right\}$ merupakan basis untuk $\left(\mathbb{F}^{B}\right)_{0}$.

Diambil sebarang elemen-elemen $a_{1}, \ldots, a_{k} \in \mathbb{F}$ sedemikian sehingga

$$
a_{1} \delta_{b_{1}}+\cdots+a_{k} \delta_{b_{k}}=0 .
$$

Diambil sebarang $b_{j} \in B$ untuk $j=1,2, \ldots, k$ diperoleh

$$
a_{1} \delta_{b_{1}}\left(b_{j}\right)+\cdots+a_{j} \delta_{b_{j}}\left(b_{j}\right)+\cdots+a_{k} \delta_{b_{k}}\left(b_{j}\right)=0+\cdots+a_{j}+\cdots+0=0 .
$$

Jadi, $a_{j}=0$ untuk $j=1,2, \ldots, k$. Dengan demikian $\mathcal{B}=\left\{\delta_{b} \mid b \in B\right\}$ bebas linier.

Selanjutnya diambil sebarang $f \in\left(\mathbb{F}^{B}\right)_{0}$. Didefinisikan $f\left(b_{s}\right)=c_{b_{s}}$. Berarti untuk sebarang $b_{j} \in B$, diperoleh

$$
\begin{aligned}
\sum_{i=1}^{k} c_{b_{i}} \delta_{b_{i}}\left(b_{j}\right) & =c_{b_{1}} \delta_{b_{1}}\left(b_{j}\right)+\cdots+c_{b_{j}} \delta_{b_{j}}\left(b_{j}\right)+\cdots+c_{b_{k}} \delta_{b_{k}}\left(b_{j}\right) \\
& =0+\cdots+c_{b_{j}}+\cdots+0 \\
& =c_{b_{j}} \\
& =f\left(b_{j}\right) .
\end{aligned}
$$

Jadi $\mathcal{B}=\left\{\delta_{b} \mid b \in B\right\}$ membangun $\left(\mathbb{F}^{B}\right)_{0}$. Dengan demikian $\mathcal{B}=\left\{\delta_{b} \mid b \in B\right\}$ membentuk basis untuk $\left(\mathbb{F}^{B}\right)_{0}$ $\operatorname{dan} \operatorname{dim}\left(\left(\mathbb{F}^{B}\right)_{0}\right)=|B|$.

Berikut diberikan definisi ruang vektor atas perluasan suatu lapangan dan operator linier pada ruang vektor tersebut.

Definisi 6. Misalkan $\mathbb{L}$ adalah suatu lapangan perluasan dari lapangan $\mathbb{F}$ dan vektor $V$ atas lapangan $\mathbb{F}$. Didefinisikan ruang vektor $V_{\mathbb{L}}=\operatorname{span}_{\mathbb{L}} V$ dan $T_{\mathbb{F}}$ adalah operator linier pada $V$ atas $\mathbb{F}$.

Pada pembahasan selanjutnya, untuk suatu polinomial $g(x) \in \mathbb{F}[x]$, kernel dari operator linier $g\left(T_{\mathbb{F}}\right)$ akan ditulis sebagai $\mathcal{S}\left(g(x), T_{\mathbb{F}}\right)$ dan notasi $T_{\mathbb{L}}$ dimaksudkan untuk suatu operator linier pada ruang vektor atas lapangan $\mathbb{L}$.

Berikut diberikan lemma tentang pembangun linier dari suatu subruang siklik dan subruang spektral dari $V$.

Lemma 7. Jika $\mathbb{L}$ adalah lapangan perluasan dari lapangan $\mathbb{F}$ maka dua pernyataan berikut benar:

1. Jika $g(x) \in \mathbb{F}[x]$, maka $\mathcal{S}\left(g(x), T_{\mathbb{L}}\right)=\operatorname{span}_{\mathbb{L}} \mathcal{S}\left(g(x), T_{\mathbb{F}}\right)$;

2. Jika $\alpha \in V_{\mathbb{F}}$, maka $Z\left(\alpha, T_{\mathbb{L}}\right)=\operatorname{span}_{\mathbb{L}} Z\left(\alpha, T_{\mathbb{F}}\right)$.

\section{Bukti.}

1. $\operatorname{span}_{\mathbb{L}} Z\left(\alpha, T_{\mathbb{F}}\right)=\left\{\sum_{i \in I} c_{i} g\left(T_{\mathbb{F}}\right)(\alpha) \mid c_{i} \in \mathbb{L}, g_{i}\left(T_{\mathbb{F}}\right) \in Z\left(\alpha, T_{\mathbb{F}}\right)\right\}$

$=\left\{\sum_{i \in I} h_{i} g\left(T_{\mathbb{L}}\right)(\alpha) \mid h_{i}(x) \in \mathbb{L}[x], \alpha \in V_{\mathbb{L}}\right\}$

$=Z\left(\alpha, T_{\mathbb{L}}\right)$

2. $\operatorname{Span}_{\mathbb{L}} \mathcal{S}\left(g(x), T_{\mathbb{F}}\right)=\operatorname{span}_{\mathbb{L}} \operatorname{Ker}\left(g\left(T_{\mathbb{F}}\right)\right)$

$$
\begin{aligned}
& =\operatorname{span}_{\mathbb{L}}\left\{v \in V_{\mathbb{F}} \mid g\left(T_{\mathbb{F}}\right)(v)=0\right\} \\
& =\left\{\sum_{i \in I} a_{i} v_{i} \mid a_{i} \in \mathbb{L}, v_{i} \in \mathcal{S}\left(g(x), T_{\mathbb{F}}\right)\right\} \\
& =\left\{u \mid g\left(T_{\mathbb{L}}\right)(u)=0\right\}
\end{aligned}
$$




$$
=\mathcal{S}\left(g(x), T_{\mathbb{L}}\right)
$$

Misalkan $V$ adalah suatu ruang vektor atas lapangan $\mathbb{L}$ dengan $\mathbb{L}$ adalah lapangan perluasan dari $\mathbb{F}$ dan $\mathfrak{A}$ adalah basis dari $V$. Sebarang automorfisma $\pi: \mathbb{L} \rightarrow \mathbb{L}$ yang membawa $\mathbb{F}$ tetap dapat diperluas menjadi pemetaan linier pada ruang vektor $\left(\mathbb{L}^{\mathfrak{2}}\right)_{0}$ yang dinotasikan dengan notasi yang sama $\pi$ dengan definisi sebagai berikut:

$$
\pi\left(\left(c_{a}\right)_{a \in \mathfrak{H}}\right)=\left(\pi\left(c_{a}\right)\right)_{a \in \mathfrak{H}}, \text { untuk setiap }\left(c_{a}\right)_{a \in \mathfrak{U}} \in\left(\mathbb{L}^{\mathfrak{N}}\right)_{0} .
$$

Lemma berikut diturunkan langsung dari paragraf sebelumnya.

Lemma 8. Jika $\pi(\lambda)=\mu$ dan $\mathbb{F}(\lambda)$ dan $\mathbb{F}(\mu)$ adalah perluasan sederhana dari $\mathbb{F}$ yang memuat $\lambda$, $\mu \in \mathbb{L}$, maka $\pi\left(V_{\mathbb{F}(\lambda)}\right)=V_{\mathbb{F}(\mu)}$ dan $\pi\left(\left(T_{\mathbb{L}}-\lambda I\right)^{m} u\right)=\left(T_{\mathbb{L}}-\mu I\right)^{m} \pi(u)$ untuk semua bilangan bulat tak-negatif $m$ dan semиa $u \in V_{\mathbb{L}}$.

Misalkan $V_{\mathbb{F}}$ adalah suatu ruang vektor atas lapangan $\mathbb{F}, T_{\mathbb{F}}$ adalah suatu operator linier pada $V_{\mathbb{F}}$ yang mempunyai polinomial minimal $m_{T_{\mathbb{F}}}(x)=p(x)^{k}$ dengan $p(x)$ adalah suatu polinomial prima di $\mathbb{F}[x]$ dan akar-akar berbeda $\lambda_{1}, \lambda_{2}, \ldots, \lambda_{n}$ di $\mathbb{L}$ dengan $\mathbb{L}$ adalah suatu lapangan perluasan dari $\mathbb{F}$ serta

$$
\rho=\left\{\begin{array}{cl}
(\operatorname{Kar}(\mathbb{F}))^{s}, & \operatorname{Kar}(\mathbb{F}) \neq 0 \\
1, & \text { lainnya }
\end{array}\right.
$$

Teorema 9. Jika $\psi(x)=p(x) /\left(x-\lambda_{i}\right)^{\rho}$ dan diberikan suatu dekomposisi siklik atau bentuk kanonik rasional dari $T$, yaitu

$$
V=\bigoplus_{\alpha \in \Lambda} Z(\alpha ; T) \operatorname{dan} T=\left.\bigoplus_{\alpha \in \Lambda} T\right|_{Z(\alpha ; T)}
$$

untuk suatu $\Lambda \subset V$, maka

1. $V_{\mathbb{K}}=\mathcal{S}\left(x-\lambda_{1} ; T_{\mathbb{K}}\right) \oplus \mathcal{S}\left(\psi(x) ; T_{\mathbb{K}}\right)$

2. $\mathcal{S}\left(x-\lambda_{1} ; T_{\mathbb{H}}\right)=\bigoplus_{\omega \in \Omega} Z\left(\omega ; T_{\mathbb{H}}\right)$, dengan $\mathbb{H}$ adalah sebarang perluasan berhingga dari lapangan $\mathbb{K}:=\mathbb{F}\left(\lambda_{1}\right)$ dan

$$
\Omega=\left\{\omega \in \mathcal{S}\left(x-\lambda_{1} ; T_{\mathbb{K}}\right) \mid \omega+\theta \in \Lambda \text { untuk suatu } \theta \in S\left(\psi(x) ; T_{\mathbb{K}}\right)\right\}
$$

3. Misalkan $\pi_{i}$ adalah automorfisma pada $\mathbb{L}$ yang membawa $\lambda=\lambda_{1}$ ke $\mu=\lambda_{i}$ dan membawa elemenelemen $\mathbb{F}$ tetap, maka $\mathbb{F}\left(\lambda_{i}\right)=\pi_{i}(\mathbb{K})$ dan untuk $i=1,2, \ldots, n$, berlaku

$$
\begin{gathered}
\mathcal{S}\left(x-\lambda_{i} ; T_{\pi_{i}(\mathbb{K})}\right)=\mathcal{S}\left(x-\lambda_{i} ; T_{\mathbb{L}}\right) \cap V_{\pi_{i}(\mathbb{K})}=\pi_{i}\left(\mathcal{S}\left(x-\lambda_{i} ; T_{\mathbb{K}}\right)\right) \\
V_{\mathbb{F}}=\left\{\bigoplus_{i=1}^{n} \pi_{i}(u) \in \bigoplus_{i=1}^{n} \mathcal{S}\left(x-\lambda_{i} ; T_{\mathbb{L}}\right) \mid u \in \mathcal{S}\left(x-\lambda_{1} ; T_{\mathbb{K}}\right)\right\}
\end{gathered}
$$

\section{Bukti.}

1. Polinomial minimal dari $T$ dapat ditulis kembali menjadi

$$
m_{T}(x)=p(x)^{k}=\left(x^{\rho}-\lambda^{\rho}\right) \psi(x)^{k}
$$

dengan $\psi(x) \in \mathbb{K}[x]$. Berdasarkan Teorema Dekomposisi Primer diperoleh

$$
V_{\mathbb{K}}=\mathcal{S}\left(x-\lambda ; T_{\mathbb{K}}\right) \oplus \mathcal{S}\left(\psi(x) ; T_{\mathbb{K}}\right) .
$$

2. Karena $\Lambda \subset V$, maka untuk masing-masing $\alpha \in \Lambda$,

$$
\alpha=\omega+\theta \in \mathcal{S}\left(x-\lambda ; T_{\mathbb{K}}\right) \oplus \mathcal{S}\left(\psi(x) ; T_{\mathbb{K}}\right),
$$

dengan $\omega$ dan $\theta$ bergantung pada $\lambda$. Kemudian dengan Lemma 7 diperoleh

$$
\begin{aligned}
V_{\mathbb{K}}=\operatorname{span}_{\mathbb{K}} V_{\mathbb{F}} & =\operatorname{span}_{\mathbb{K}} \bigoplus_{\alpha \in \Lambda} Z\left(\alpha ; T_{\mathbb{F}}\right)=\oplus_{\alpha \in \Lambda} Z\left(\alpha ; T_{\mathbb{K}}\right) \\
& =\bigoplus_{\alpha \in \Lambda} Z\left(\omega+\theta ; T_{\mathbb{K}}\right) \\
& =\left(\bigoplus_{\alpha \in \Lambda} Z\left(\omega ; T_{\mathbb{K}}\right)\right) \oplus\left(\oplus_{\alpha \in \Lambda} Z\left(\theta ; T_{\mathbb{K}}\right)\right) \\
& \subset \mathcal{S}\left(x-\lambda_{1} ; T_{\mathbb{K}}\right) \bigoplus \mathcal{S}\left(\psi(x) ; T_{\mathbb{K}}\right)=V_{\mathbb{K}} .
\end{aligned}
$$

Kemudian 


$$
\mathcal{S}\left(x-\lambda ; T_{\mathbb{K}}\right)=\bigoplus_{\omega \in \Omega} Z\left(\omega ; T_{\mathbb{K}}\right)
$$

dan

$$
\mathcal{S}\left(x-\lambda ; T_{\mathbb{H}}\right)=\operatorname{span}_{\mathbb{H}} \mathcal{S}\left(x-\lambda ; T_{\mathbb{K}}\right)=\operatorname{span}_{\mathbb{H}} \bigoplus_{\omega \in \Omega} Z\left(\omega ; T_{\mathbb{K}}\right)=\bigoplus_{\omega \in \Omega} Z\left(\omega ; T_{\mathbb{H}}\right) .
$$

3. Berdasarkan Lemma 8 diperoleh

$$
\begin{aligned}
\mathcal{S}\left(x-\lambda_{i} ; T_{\pi_{i}(\mathbb{K})}\right) & =\mathcal{S}\left(x-\lambda_{i} ; T_{\mathbb{L}}\right) \cap V_{\pi_{i}(\mathbb{K})}=\pi_{i}\left(\mathcal{S}\left(x-\lambda_{1} ; T_{\mathbb{L}}\right)\right) \cap \pi_{i}\left(V_{\mathbb{K}}\right) \\
& =\pi_{i}\left(\mathcal{S}\left(x-\lambda_{1} ; T_{\mathbb{L}}\right) \cap\left(V_{\mathbb{K}}\right)\right) \\
& =\pi_{i}\left(\mathcal{S}\left(x-\lambda_{1} ; T_{\mathbb{K}}\right)\right)
\end{aligned}
$$

dengan $T_{\pi_{i}(\mathbb{K})}=\left.T_{\mathbb{L}}\right|_{V_{\pi_{i}(\mathbb{K})}}$ untuk $i=1,2, \ldots, n$. Selanjutnya misalkan $j \in\{1,2, \ldots, n\}$ dan $v \in V_{\mathbb{F}}$. Diperhatikan

$$
v=\bigoplus_{i=1}^{n} u_{i} \in \bigoplus_{i=1}^{n} \mathcal{S}\left(x-\lambda_{i} ; T_{\mathbb{L}}\right)
$$

dan

$$
v=\pi_{j}(v)=\pi_{j}\left(\sum_{i=1}^{n} u_{i}\right)=\sum_{i=1}^{n} \pi_{j}\left(u_{i}\right)
$$

Berdasarkan Lemma $8, \pi_{j}\left(u_{1}\right)$ dan $u_{j}$ berada di $\delta\left(x-\lambda_{j} ; T_{\mathbb{L}}\right)$. Untuk membuktikan persamaan terakhir, sama halnya dengan menunjukkan bahwa $\pi_{j}\left(u_{1}\right)=u_{j}$ untuk setiap $j=1,2, \ldots, n$. Dengan kata lain, akan ditunjukkan bahwa $\pi_{j}\left(u_{i}\right) \notin \mathcal{S}\left(x-\lambda_{j} ; T_{\mathbb{L}}\right)$ untuk $i \neq j$. Untuk tiap-tiap $i \in$ $\{1,2, \ldots, n\}$, terdapat $r=r(i) \in\{1,2, \ldots, n\}$ sedemikian sehingga $\pi_{j}\left(u_{i}\right) \notin \mathcal{S}\left(x-\lambda_{r} ; T_{\mathbb{L}}\right)$.

Diklaim bahwa pemetaan $i \mapsto r(i)$ adalah pemetaan bijektif.

Diandaikan bahwa pemetaan $i \mapsto r(i)$ bukan pemetaan bijektif, maka terdapat $t \in$ $\{1,2, \ldots, n\}$ sedemikian sehingga

$$
v \in \bigoplus_{i \neq t} \mathcal{S}\left(x-\lambda_{i} ; T_{\mathbb{L}}\right)
$$

dan $q(T)(v)=q\left(T_{\mathbb{L}}\right)(v)=0$ untuk suatu polinomial $q(x) \in \mathbb{L}[x]$ yang tidak terbagi oleh $x-\lambda_{t}$. Karena $v \in V, T \in L(V)$ dan $p(T)^{k}(v)=0$, akibatnya $q(x) \in \mathbb{F}[x]$ dan $q(x) \mid p(x)^{k}$. Hal ini kontradiksi dengan $p(x)^{k}$ adalah polinomial minimal dari $T$. Jadi, haruslah pemetaan

$$
i \mapsto r(i)
$$

bijektif, yaitu untuk tiap-tiap $i \in\{1,2, \ldots, n\}$ terdapat $r=r(i)$ sedemikian sehingga $\pi_{j}\left(u_{i}\right) \in$ $\mathcal{S}\left(x-\lambda_{j} ; T_{\mathbb{L}}\right)$, untuk $i \neq j$ dan $\pi_{j}\left(u_{1}\right) \in \mathcal{S}\left(x-\lambda_{j} ; T_{\mathbb{L}}\right)$.

Berikut akan diberikan definisi dari basis dekomposisi Jordan dan basis dekomposisi siklik dari $T$.

Misalkan $T$ adalah suatu operator linier pada ruang vektor $V_{\mathbb{F}}$ dan

$$
V=\bigoplus_{\alpha \in \Lambda} Z(\alpha ; T)
$$

adalah dekomposisi siklik dari $V_{\mathbb{F}}$ terhadap $T$ dan $\mathbb{L}$ adalah perluasan berhingga dari lapangan $\mathbb{F}$ yang berisi semua akar-akar $\lambda_{1}, \lambda_{2}, \ldots, \lambda_{n}$ dari polinomial minimal $m_{T}(x)=p(x)^{k}$ dari $T$.

Definisi 10. Himpunan $\Lambda \subset V$ pada bentuk (7) disebut basis dekomposisi siklik atau basis bentuk kanonik rasional dari $T$ sedangkan himpunan $\Gamma=\mathrm{U}_{i=1}^{n} \Omega_{i}$ disebut basis dekomposisi Jordan atau basis bentuk kanonik Jordan dari $T_{\mathbb{L}} j i k a \mathcal{S}\left(x-\lambda_{i} ; T_{\mathbb{L}}\right)=\bigoplus_{\omega \in \Omega_{i}} Z\left(\omega ; T_{\mathbb{L}}\right)$ dengan $\lambda_{1}, \lambda_{2}, \ldots, \lambda_{n}$ adalah nilai eigen yang berbeda dari $T_{\mathbb{L}}$.

Selain itu basis Jordan $\Gamma=\cup_{i=1}^{n} \Omega_{i}$ juga dikenal sebagai basis $T_{\mathbb{L}}$-siklik primer dari $T$ karena dalam memperoleh basis tersebut menggunakan dekomposisi siklik dan dekomposisi primer pada $V$ terhadap $T_{\mathbb{L}}$. 
Diasumsikan bahwa $T_{\mathbb{F}} \in L\left(V_{\mathbb{F}}\right)$ mempunyai polinomial minimal $m_{T_{\mathbb{F}}}(x)=p(x)^{k}$ dengan akar-akar $\lambda_{1}, \lambda_{2}, \ldots, \lambda_{n}$ di suatu lapangan perluasan berhingga $\mathbb{L}$ dari $\mathbb{F}$. Misalkan $\pi_{i}$ adalah suatu automorfisma dari lapangan $\mathbb{L}$ yang membawa $\lambda_{1}$ ke $\lambda_{i}(i=1,2, \ldots, n)$ dan membawa elemen-elemen $\mathbb{F}$ tetap. Misalkan $\Gamma=$ $\cup_{i=1}^{n} \Omega_{i}$ memenuhi

$$
\mathcal{S}\left(x-\lambda_{i} ; T_{\mathbb{L}}\right)=\oplus_{\omega \in \Omega_{i}} Z\left(\omega ; T_{\mathbb{L}}\right), \text { untuk } i=1,2, \ldots, n .
$$

Teorema 11. Basis dekomposisi Jordan $\Gamma=\cup_{i=1}^{n} \Omega_{i}$ dari $T_{\mathbb{L}}$ dapat diubah menjadi basis dekomposisi siklik $\Lambda$ dari $T_{\mathbb{F}}$ dengan setiap $\alpha \in \Lambda$ berbentuk $\alpha=\omega_{1} \oplus \omega_{2} \oplus \ldots \oplus \omega_{n}$ untuk suatu $\omega_{i} \in \Omega_{i}(i=1,2, \ldots, n)$ jika dan hanya jika $\Gamma=\cup_{i=1}^{n} \Omega_{i}$ memenuhi kondisi-kondisi berikut:

1. $\Omega_{1}$ berada di $\mathcal{S}\left(x-\lambda_{1} ; T_{\mathbb{F}\left(\lambda_{1}\right)}\right)$.

2. $\Omega_{i}=\pi_{i}\left(\Omega_{1}\right)$ untuk $i=1,2, \ldots, n$.

3. $\sum_{i=1}^{n} \pi_{i}(\omega) \in V_{\mathbb{F}}$ untuk semua $\omega \in \Omega_{1}$.

Bukti. $(\Rightarrow)$ Bagian ini dapat dibuktikan dengan menggunakan Teorema 9. $(\Leftarrow)$ Selanjutnya diasumsikan poin 1,2, dan 3 terpenuhi. Akan ditunjukkan bahwa basis dekomposisi Jordan $\Gamma=\cup_{i=1}^{n} \Omega_{i}$ dapat diubah menjadi basis dekomposisi siklik $\Lambda$. Didefinisikan

$$
\Lambda=\left\{\bigoplus_{i=1}^{n} \pi_{i}(\omega): \omega \in \Omega_{1}\right\}
$$

Berdasarkan poin 3, berarti $\Lambda \subset V_{\mathbb{F}}$. Dengan Lemma 7, diperoleh

$$
\begin{aligned}
\operatorname{span}_{\mathbb{L}} V_{\mathbb{F}}=V_{\mathbb{L}} & =\bigoplus_{i=1}^{n} \mathcal{S}\left(x-\lambda_{i} ; T_{\mathbb{L}}\right) \\
& =\bigoplus_{i=1}^{n} \bigoplus_{\omega \in \Omega_{1}} Z\left(\omega, T_{\mathbb{L}}\right) \\
& =\bigoplus_{i=1}^{n} \bigoplus_{\omega \in \Omega_{1}} Z\left(\pi_{i}(\omega), T_{\mathbb{L}}\right) \\
& =\bigoplus_{\omega \in \Omega_{1}} \bigoplus_{i=1}^{n} Z\left(\pi_{i}(\omega), T_{\mathbb{L}}\right) \\
& =\bigoplus_{\omega \in \Omega_{1}} Z\left(\bigoplus_{i=1}^{n} \pi_{i}(\omega), T_{\mathbb{L}}\right) \\
& =\bigoplus_{\alpha \in \Lambda} Z\left(\alpha, T_{\mathbb{L}}\right) \\
& =\operatorname{span}_{\mathbb{L}} \bigoplus_{\alpha \in \Lambda} Z\left(\alpha, T_{\mathbb{F}}\right)
\end{aligned}
$$

Karena $Z\left(\alpha, T_{\mathbb{F}}\right) \subset V_{\mathbb{F}}$, berarti $V_{\mathbb{F}}=\bigoplus_{\alpha \in \Lambda} Z\left(\alpha ; T_{\mathbb{F}}\right)$.

Definisi 12. Dekomposisi Jordan atau lebih khususnya basis dekomposisi Jordan $\Gamma$ dikatakan simetris jika bagian-bagiannya $\Omega_{\mathrm{i}}$ memenuhi kondisi 1) dan 2) pada Teorema 11.

Teorema berikut menunjukkan bahwa jika suatu operator linier $T$ mempunyai basis dekomposisi Jordan $\Gamma$ yang simetris, maka basis $\Gamma$ tersebut akan memenuhi poin 1) dan 2) dari Teorema 11.

Teorema 13. Jika $T_{\mathbb{F}}$ adalah operator linier pada ruang vektor $V_{\mathbb{F}}$ yang mempunyai polinomial minimal $m_{T_{\mathbb{F}}}(x)=p(x)^{k}$ untuk suatu polinomial prima $p(x) \in \mathbb{F}[x]$ yang terfaktorkan secara lengkap di lapangan $\mathbb{L}$ dari $m_{T_{\mathbb{F}}}(x)$ maka $T_{\mathbb{L}}$ mempunyai suatu dekomposisi Jordan dengan basis yang simetris $\Gamma=\cup_{i=1}^{n} \Omega_{i}$ yang dibangun oleh $\Omega$.

Bukti. Misal $\mathbb{K}=\mathbb{F}\left(\lambda_{1}\right)$ adalah lapangan perluasan berhingga dari $\mathbb{F}$. Untuk menemukan basis simetris $\Gamma=$ $\cup_{i=1}^{n} \Omega_{1}$ untuk dekomposisi Jordan dari $T_{\mathbb{L}}$, misalkan $\Omega$ adalah basis dari sebarang dekomposisi Jordan dari suatu operator nilpoten $\left.\left.\left(T_{\mathbb{K}}-\lambda_{i}\right)\right|_{\mathcal{S}\left(x-\lambda_{1}, T_{\mathbb{K}}\right.}\right)$. Yaitu $\mathcal{S}\left(x-\lambda_{1} ; T_{\mathbb{K}}\right)=\bigoplus_{\omega \in \Omega} Z\left(\omega ; T_{\mathbb{K}}-\lambda_{1} I\right)=$ $\bigoplus_{\omega \in \Omega} Z\left(\omega ; T_{\mathbb{K}}\right)$. Misalkan $\Gamma=\cup_{i=1}^{n} \Omega_{1}$ (dengan $\pi_{1}$ adalah fungsi identitas) untuk $i=1,2, \ldots, n$. Berdasarkan Lemma 7 dan Lemma 8, diperoleh

$$
\begin{aligned}
\mathcal{S}\left(x-\lambda_{i} ; T_{\mathbb{L}}\right) & =\pi_{i}\left(\mathcal{S}\left(x-\lambda_{1} ; T_{\mathbb{L}}\right)\right) \\
& =\pi_{i}\left(\operatorname{span}_{\mathbb{L}} \mathcal{S}\left(x-\lambda_{1} ; T_{\mathbb{K}}\right)\right) \\
& =\pi_{i}\left(\operatorname{span}_{\mathbb{L}} \bigoplus_{\omega \in \Omega_{1}} Z\left(\omega ; T_{\mathbb{K}}\right)\right)
\end{aligned}
$$




$$
\begin{aligned}
& =\pi_{i}\left(\oplus_{\omega \in \Omega_{1}} Z\left(\omega ; T_{\mathbb{L}}\right)\right) \\
& =\bigoplus_{\omega \in \Omega_{1}} Z\left(\pi_{i}(\omega) ; T_{\mathbb{L}}\right)
\end{aligned}
$$

Berikut akan diberikan beberapa contoh terkait dengan perubahan basis dekomposisi Jordan menjadi basis dekomposisi siklik.

Contoh 14. Diberikan operator linier $T$ pada ruang vektor $\mathbb{Q}^{2}$ dengan polinomial karakteristik dari $T$ yang diperoleh dari bentuk kanonik Jordannya adalah $p_{T}(x)=x^{2}-2 x+5$. Nilai eigen dari $T$ adalah $\lambda_{1}=1-$ $2 i$ dan $\lambda_{2}=1+2 i$ dengan vektor eigen yang bersesuaian adalah $\left[\begin{array}{c}1 \\ 1+i\end{array}\right]$ dan $\left[\begin{array}{c}1 \\ 1-i\end{array}\right]$. Diperoleh

$$
\Omega_{1}=\left\{\left[\begin{array}{c}
1 \\
1+i
\end{array}\right]\right\} \subset \mathcal{S}\left(x-(1-2 i), T_{\mathbb{Q}(1-2 i)}\right) \text {. }
$$

Diberikan suatu $\mathbb{C}$-automorfisma $\pi_{i}: \mathbb{C} \rightarrow \mathbb{C}$, dengan $\pi_{i}: \mathbb{Q} \rightarrow \mathbb{Q}$ dan $\pi_{i}: \lambda_{1} \mapsto \lambda_{i}$ untuk $i=1,2$. Berarti $\pi_{1}(i)=i$ dan $\pi_{2}(i)=-i$. Diperhatikan

$$
\begin{gathered}
\pi_{1}\left(\left[\begin{array}{c}
1 \\
1+i
\end{array}\right]\right)=\left[\begin{array}{c}
\pi_{1}(1) \\
\pi_{1}(1+i)
\end{array}\right]=\left[\begin{array}{c}
1 \\
1+i
\end{array}\right] \\
\pi_{2}\left(\left[\begin{array}{c}
1 \\
1+i
\end{array}\right]\right)=\left[\begin{array}{c}
\pi_{2}(1) \\
\pi_{2}(1+i)
\end{array}\right]=\left[\begin{array}{c}
1 \\
1-i
\end{array}\right] \in \mathcal{S}\left(x-(1+2 i), T_{\mathbb{Q}(1+2 i)}\right) .
\end{gathered}
$$

Diperoleh

$$
\pi_{1}\left(\left[\begin{array}{c}
1 \\
1+i
\end{array}\right]\right)+\pi_{2}\left(\left[\begin{array}{c}
1 \\
1+i
\end{array}\right]\right)=\left[\begin{array}{c}
1 \\
1+i
\end{array}\right]+\left[\begin{array}{c}
1 \\
1-i
\end{array}\right]=\left[\begin{array}{l}
2 \\
2
\end{array}\right] \in \mathbb{Q}^{2}
$$

Berdasarkan Teorema 10, operator linier $T$ mempunyai suatu basis siklik atas lapangan $\mathbb{Q}$. Lebih lanjut, $T\left(\left[\begin{array}{c}1 \\ 1+i\end{array}\right]\right)=\left[\begin{array}{ll}3 & -2 \\ 4 & -1\end{array}\right]\left[\begin{array}{l}2 \\ 2\end{array}\right]=\left[\begin{array}{l}2 \\ 6\end{array}\right]$. Dapat ditunjukkan bahwa himpunan $\mathcal{A}=\left\{\left[\begin{array}{l}2 \\ 2\end{array}\right],\left[\begin{array}{l}2 \\ 6\end{array}\right]\right\}$ membentuk basis untuk $\mathbb{Q}^{2}$. Dengan demikian, $\mathbb{Q}^{2}=Z\left(\left[\begin{array}{l}2 \\ 2\end{array}\right], T_{\mathbb{Q}}\right)$ dan $p_{T}(x)=x^{2}-2 x+5$ adalah polinomial $\mathbb{Q}$-annihilator dari vektor $\left[\begin{array}{l}2 \\ 2\end{array}\right]$. Jadi, matriks bentuk kanonik rasional dari $T$ atas lapangan $\mathbb{Q}$ adalah $R_{T}=\left[\begin{array}{cc}0 & -5 \\ 1 & 2\end{array}\right]$.

Contoh 15. Diberikan operator linier $T$ pada ruang vektor $\mathbb{Q}^{2}$ dengan nilai eigen dari $T$ yang diperolah dari matriks kanonik Jordannya adalah $\lambda_{1}=-4+2 i, \lambda_{2}=-4-2 i, \lambda_{3}=2+3 i$, dan $\lambda_{4}=2-3 i$ dengan vektor eigen yang bersesuaian adalah

$$
\left[\begin{array}{l}
1 \\
i \\
0 \\
0
\end{array}\right],\left[\begin{array}{c}
1 \\
-i \\
0 \\
0
\end{array}\right],\left[\begin{array}{l}
0 \\
0 \\
1 \\
i
\end{array}\right],\left[\begin{array}{c}
0 \\
0 \\
1 \\
-i
\end{array}\right] .
$$

Diberikan $\mathbb{C}$-automorfisma $\pi_{i}: \mathbb{C} \rightarrow \mathbb{C}$, dengan $\pi_{i}(\mathbb{Q})=\mathbb{Q}$ dan $\pi_{i}\left(\lambda_{1}\right)=\lambda_{1}$ untuk $i=1,2,3,4$. Berarti $\pi_{1}(i)=i, \pi_{2}(i)=-i, \pi_{3}(i)=3+\frac{3}{2} i$, dan $\pi_{4}(i)=3-\frac{3}{2} i$. Diperoleh

$$
\pi_{2}\left(\left[\begin{array}{l}
1 \\
i \\
0 \\
0
\end{array}\right]\right)=\left[\begin{array}{c}
1 \\
-i \\
0 \\
0
\end{array}\right] \in E\left(\lambda_{2}\right), \quad \pi_{3}\left(\left[\begin{array}{l}
1 \\
i \\
0 \\
0
\end{array}\right]\right)=\left[\begin{array}{c}
1 \\
3+\frac{3}{2} i \\
0 \\
0
\end{array}\right] \notin E\left(\lambda_{3}\right), \quad \pi_{4}\left(\left[\begin{array}{l}
1 \\
i \\
0 \\
0
\end{array}\right]\right)=\left[\begin{array}{c}
1 \\
3-\frac{3}{2} i \\
0 \\
0
\end{array}\right] \notin E\left(\lambda_{4}\right)
$$

Berdasarkan Definisi 12, himpunan 


$$
\left\{\left[\begin{array}{l}
1 \\
i \\
0 \\
0
\end{array}\right],\left[\begin{array}{c}
1 \\
-i \\
0 \\
0
\end{array}\right],\left[\begin{array}{l}
0 \\
0 \\
1 \\
i
\end{array}\right],\left[\begin{array}{c}
0 \\
0 \\
1 \\
-i
\end{array}\right]\right\}
$$

bukan merupakan basis simetris dari dekomposisi Jordan dari $T$, sehingga berlum terjamin dapat dibentuk basis dekomposisi siklik dari $T$ dengan menggunakan basis Jordan (8).

Kadangkala proses yang diuraikan pada teorema sebelumnya tidak selalu berlaku, sehingga untuk memperoleh basis siklik dari $T$ bisa menggunakan cara langsung yaitu dengan menjumlahkan beberapa vektor basis Jordan dari $T$.

Contoh 16. Diberikan operator linier $T$ poada ruang vektor $\mathbb{R}^{3}$ dengan matriks bentuk kanonik Jordannya adalah

$$
\left[\begin{array}{ccc}
2 & 0 & 0 \\
0 & 2+3 i & 0 \\
0 & 0 & 2-3 i
\end{array}\right]
$$

Nilai eigen dari $T$ adalah $\lambda_{1}=2, \lambda_{2}=2+3 i$ dan $\lambda_{3}=2-3 i$ di lapangan $\mathbb{C}$, dengan vektor eigen yang bersesuai

$$
w_{1}=\left[\begin{array}{l}
1 \\
0 \\
0
\end{array}\right], w_{2}=\left[\begin{array}{c}
0 \\
-i \\
1
\end{array}\right], \text { dan } w_{3}=\left[\begin{array}{l}
0 \\
i \\
1
\end{array}\right]
$$

Dapat ditunjukkan bahwa $\mathcal{B}=\left\{w_{1}, w_{2}, w_{3}\right\}$ membentuk basis untuk ruang vektor $\mathbb{C}^{3}$. Akibatnya, $\mathbb{C}^{3}$ dapat didekomposisikan menjadi

$$
\mathbb{C}^{3}=Z\left(w_{1} ; T_{\mathbb{C}}\right) \oplus Z\left(w_{2} ; T_{\mathbb{C}}\right) \oplus Z\left(w_{3} ; T_{\mathbb{C}}\right)
$$

Diperoleh

$$
\begin{aligned}
\operatorname{span}_{\mathbb{C}} \mathbb{R}^{3}=\mathbb{C}^{3} & =Z\left(w_{1} ; T_{\mathbb{C}}\right) \bigoplus Z\left(w_{2} ; T_{\mathbb{C}}\right) \oplus Z\left(w_{3} ; T_{\mathbb{C}}\right)=Z\left(w_{1}+w_{2}+w_{3} ; T_{\mathbb{C}}\right) \\
& =Z\left(\left[\begin{array}{l}
1 \\
0 \\
0
\end{array}\right]+\left[\begin{array}{c}
0 \\
-i \\
1
\end{array}\right]+\left[\begin{array}{l}
0 \\
i \\
1
\end{array}\right] ; T_{\mathbb{C}}\right) \\
& =\operatorname{span}_{\mathbb{C}} Z\left(\left[\begin{array}{l}
1 \\
0 \\
2
\end{array}\right] ; T_{\mathbb{R}}\right)
\end{aligned}
$$

Karena $\mathbb{R}^{3}$ dan $Z\left(\left[\begin{array}{l}1 \\ 0 \\ 2\end{array}\right] ; T_{\mathbb{R}}\right)$ adalah subruang linier dari $\mathbb{R}^{3}$, akibatnya

$$
\mathbb{R}^{3}=Z\left(\left[\begin{array}{l}
1 \\
0 \\
2
\end{array}\right] ; T_{\mathbb{R}}\right)
$$

Yang memberikan bentuk kanonik rasional dari $T$ sebagai berikut:

$$
\left[\begin{array}{ccc}
0 & 0 & 26 \\
1 & 0 & -2 \\
0 & 1 & 6
\end{array}\right]
$$




\section{KESIMPULAN}

Dari uraian pada bagian sebelumnya, dapat ditarik kesimpulan, sebagai berikut:

1. Pembentukan matriks bentuk kanonik rasional $R_{T}$ dari suatu operator linier $T$ menggunakan dekomposisi siklik ruang vektor, sedangkan pembentukan matriks bentuk kanonik Jordannya menggunakan dekomposisi primer dan dekomposisi siklik ruang vektor.

2. Matriks kanonik rasional $R_{T}$ dari $T$ tidak bergantung kepada polinomial karakteristik $p_{T}(x)$ dan polinomial minimal $m_{T}(x)$ dari $T$, artinya dalam keadaan apapun, matriks kanonik rasional $R_{T}$ dari $T$ selalu dapat dibentuk.

3. Matriks kanonik Jordan $J_{T}$ dari $T$ sangat bergantung kepada polinomial karakteristik $p_{T}(x)$ dan polinomial minimal $m_{T}(x)$ dari $T$, artinya ketika $p_{T}(x)$ tidak terfaktorkan secara lengap dilapangan skalarnya, $J_{T}$ tidak dapat dibentuk.

4. Ketika polinomial karakteristik $p_{T}(x)$ dari $T$ terfaktorkan secara lengkap di lapangan skalar dari $T$, matriks $R_{T}$ dapat diubah menjadi matriks $J_{T}$, demikian pula sebaliknya atas lapangan yang sama.

5. Ketika polinomial karakteristik $p_{T}(x)$ dari $T$ tidak terfaktorkan secara lengkap di lapangan skalar dari $T$, matriks $R_{T}$ tetap dapat diubah menjadi matriks $J_{T}$ atas lapangan perluasan, namun perubahan matriks $J_{T}$ atas lapangan perluasan menjadi matriks $R_{T}$ atas lapangan asal mengunakan simetrisasi pada basis Jordan dari $T$, yaitu dengan mengubah basis Jordan yang simetris menjadi basis siklik dari $T$, sehingga diperoleh dekomposisi siklik dari $V$ untuk membentuk matriks kanonik rasioanl $R_{T}$.

\section{DAFTAR PUSTAKA}

[1] J. B. Fraleigh, A First Course in Abstract Algebra, Seventh Edition, New York: Addison Wesley, 2003.

[2] Kenneth Hoffman, Ray Kunze, Linear Algebra, New Jersey: Prentice-Hall, Inc, Engliwood Cliffts, 1971.

[3] M. Radjabalipour, "The Rational Canonical Form Via The Splitting Field", Journal of Linear Algebra and Its Applications, pp. 2250-2255, July 2013.

[4] M. Radjabalipour, "A Symmetrization of The Jordan Canonical Form", Journal of Linear Algebra and Its Applications, pp. 94-112, 2017.

[5] S. H. Weintraub, Jordan Canonical Form, Theory and Practice, Washington: Morgan and Claypool Publisher, 2009. 
